\title{
CAMBIAR PARA REFORMAR: NUEVOS LINEAMIENTOS CURRICULARES PARA LA FORMACIÓN DE PSICÓLOGOS EN SAN MARCOS
}

\author{
Lupe Garcia A. ${ }^{1}$, Oswaldo Orellana M. ${ }^{2}$, Jaime Aliaga T. ${ }^{3}$, Roger Elizalde B. ${ }^{4}$, José Luis Perea R. ${ }^{5}$
}

(Recibido el 02/11/2006, aceptado el 30/11/2006)

\section{RESUMEN}

Expone las conclusiones y recomendaciones del Seminario curricular 2006, que concluye en la reforma curricular para la formación de psicólogos en la Universidad Nacional Mayor de San Marcos. El evento se llevó a cabo en los meses de mayo, junio, julio y agosto, orientado bajo la orientación de la visión y misión planteada por una «Comisión transitoria». La visión planteada tuvo el siguiente contenido, «lograr un sistema curricular para la formación profesional universitaria del psicólogo, que viabilice y garantice el camino hacia la acreditación universitaria en los ámbitos nacional e internacional», y la misión, «cumplir con los requerimientos que exige la excelencia académica, desarrollando una gestión curricular eficiente, que permita el cambio para una formación universitaria, de calidad profesional que responda a las expectativas de la comunidad psicológica y la nación peruana».

Palabras clave: Producción de conocimientos, Currículo universitario, Acreditación de la calidad, Formación académica, Reforma curricular.

\begin{abstract}
It exposes the conclusions and recommendations of the Curricular Seminary 2006, that concludes in the curricular reform for formation of psychologist in Universidad Nacional Mayor de San Marcos. The event was carried out in the months of May, June, July and August, oriented under orientacin of vision and mission raised by one Comision Transitory. The raised vision had the following content, «to obtain a curricular system for the university professional formation of the psychologist, who viabilice and guarantees the way towards the university accreditation in the scopes national and international», and the mission, «to fulfill the requirements that the academic excellence demands, developing an efficient curricular management, that allows the change for a university formation, of professional quality that responds to the expectations of the psychological community and the Peruvian nation».
\end{abstract}

Keywords: Production of knowledge, University currículo, Accreditation of the quality, academic Formation, Curricular reformation.

\footnotetext{
Docente Principal y Decana de la Facultad de Psicología de la UNMSM. E-mail: Igarciape@yahoo.es

2 Docente Principal de la Facultad de Psicología de la UNMSM. E-mail: ovaorellana@yahoo.es

Docente Principal de la Facultad de Psicología de la UNMSM. E-mail: alaito@terra.com

4 Docente Principal de la Facultad de Psicología de la UNMSM. E-mail: relizaldeb@unmsm.edu.pe

5 Docente Auxiliar de la Facultad de Psicología de la UNMSM. E-mail: jperear@unmsm.edu.pe
} 


\section{INTRODUCCIÓN}

La apertura al debate académico, sistemático, alturado y con bases científicas y doctrinarias, caracterizó al seminario curricular 2006. Estas se expresaron en cinco jornadas organizadas en los meses de mayo, junio, julio y agosto, bajo la orientación de un programa sucesivo de temas centrales selectos, permitiendo madurar y construir una alternativa de reforma curricular en la Facultad de Psicología.

En algunos momentos de la historia universitaria, se experimenta la fluidez de las ideas innovadoras y el apasionamiento de su defensa, y esto sucedió en San Marcos, como algo típico de su quehacer cotidiano, pues la caracteriza, define y mantiene su vigencia en la vida académica. La oportuna programación inicial de los temas sobre calidad académica, la autoevaluación para la mejora, la enseñanza universitaria y la elaboración de los perfiles, permitieron tomar conciencia que nos encontramos en un cambio de época, donde la descontextualización de las prácticas anteriores, es la urgencia en la línea que traza el desarrollo de la enseñanza universitaria. Siendo imperativo que la comunidad universitaria ponga todos sus esfuerzos para avanzar y colocarnos a la altura de las exigencias nacionales e internacionales.

El aporte del Comité Asesor de la EAP de Psicología fue decisivo en el ordenamiento del debate, ya que el documento titulado «Sistema curricular de la Facultad de Psicología» fue el instrumento orientador como «términos de referencia» y guía para el debate en la búsqueda de alternativas de reforma.

Otro hecho importante ha sido la participación de docentes y estudiantes, en grupos mixtos, que bien pueden tipificarse como la gran avanzada en las inquietudes académicas y profesionales. De esta forma, las secciones como organizaciones académicas naturales de los docentes, deben constituirse en los verdaderos núcleos operativos y de vigilancia epistemológica, en la implementación de los planteamientos curriculares de cambio y reforma, de los sistemas de enseñanza y aprendizaje

La organización del seminario curricular tuvo una convocatoria mayoritaria en los docentes y significativa en los estudiantes, implementada bajo las responsabilidades estatutarias siguientes: Decanato, Consejo de Facultad, Dirección de la EAP de Psicología, Comité Asesor de la EAP de Psicología y Comisión transitoria del Seminario Curricular 2006.

El siguiente es una parte del documento mayor, referida a las conclusiones y recomendaciones (15) del Informe presentado al Consejo de Facultad, que trascribimos a continuación, por constituir un documento vital para el mejoramiento de la formación profesional de psicólogos en San Marcos:

\section{Promover una universidad que busca la verdad}

La esencia histórica de la universidad es ser una organización académica de alta calificación de producción y validación de conocimientos, donde se forman los cuadros profesionales, en un campo especifico del conocimiento científico y tecnológico, con una formación integral humanística, con conciencia ciudadana y compromiso con las exigencias históricas de la época.

La tarea actual es crear las condiciones para vincular nuestra universidad a las dimensiones nacionales e internacionales que afiance su integración a la mundializacion y a la movilidad docente y estudiantil. 


\section{Impulsar una enseñanza especializada, critica e innovadora}

La enseñanza universitaria de psicología promoverá el paradigma de la universidad como búsqueda de la verdad, trazando su hegemonía sobre los otros paradigmas.

Es un compromiso permanente mantener el debate sobre las utopías que orientan la formación profesional del psicólogo, principalmente aquellas referidas a la mente y a la conducta humana.

Organizar la enseñanza de acuerdo a los contenidos del aprendizaje: conceptual, procedimental y actitudinal, teniendo en cuenta la naturaleza de la asignatura y creando los mecanismos y criterios para valorizarlos en los docentes y los estudiantes.

La gestión de la enseñanza a través de los contenidos de aprendizaje deberá estar expresada en los sílabos, bajo el monitoreo de las secciones y dirección del coordinador del departamento. El contenido conceptual debe expresarse en conceptos, hechos y principios. Los conceptos científicos deben referirse a los atributos que contienen los objetos, situaciones o entes manifiestos en símbolos y signos organizados, para comprender un hecho concreto o una formulación abstracta. Los hechos referidos a los eventos que se registran en la historia social o individual de los sujetos deben permitir entender una dimensión de la individualidad y de la sociedad. Los principios referidos a las proposiciones derivadas de afirmaciones previas de objetos abstractos o concretos deben abordar la vigencia o no vigencia de una teoría.

Los contenidos procedimentales referidos a los algoritmos y los heurísticos, así mismo a los procedimientos, estrategias y técnicas, deben permitir que el alumno construya un conocimiento en base a secuencias establecidas por la naturaleza del objeto, organizando la información para un mejor conocimiento y toma de decisiones consecuentes

Los contenidos actitudinales referidos a los valores, normas y actitudes positivas, adquiridas en la relación profesor-alumno, deben definir los valores que se promoverían en las asignaturas, evitando que el currículo oculto hegemonice las prácticas docentes en el aula.

El Departamento de Psicología, conjuntamente con la Escuela Académico Profesional, deberá definir los tipos de asignaturas y las estrategias de enseñanza adecuadas, empleando criterios de las modernas teorías del aprendizaje o sustentar los métodos clásicos que todavía se manifiestan: clases magistrales, seminarios, talleres, entre otros.

Fortalecer las secciones del departamento académico de psicología como organización natural de los docentes y núcleos de trabajo especializado del conocimiento psicológico y principal soporte activo para la enseñanza universitaria.

Valorizar adecuadamente el método experimental en la enseñanza, así mismo los métodos cuantitativos y cualitativos, en su pertinencia formativa.

La EAP de psicología establecerá la política de desarrollo de la enseñanza experimental, definiendo las asignaturas involucradas y la promoción de actividades de tal naturaleza.

En cada ciclo es tarea de la EAP de Psicología definir los contenidos transversales que los docentes deben desarrollar en el contenido de las asignaturas a su cargo. Estos 
contenidos deben concretar tópicos de la interculturalidad, el género, la ética, entre otros.

La innovación y el cambio deberá trazar el modo de construcción de conocimientos, orientado por la interdisciplinariedad en las dimensiones nacionales de multiculturalidad y multilingüística.

\section{Mantener la misión de San Marcos como promotora de la psicología en el Perú}

San Marcos, como universidad fundadora de la formación de psicólogos en el Perú, asumió el compromiso y la responsabilidad de orientar el conocimiento y el entrenamiento académico y profesional, a través de una educación integral, humanística y especializada en los niveles de pre y posgrado.

\section{Asumir la meta de la acreditación de la calidad}

La comunidad universitaria de psicología asume el compromiso de trazar la meta de lograr la acreditación de la calidad. La calidad universitaria, como rendición de cuentas al país sobre el cumplimiento de las funciones inherentes a su naturaleza, debe responder a las necesidades que demanda la realidad nacional, contribuyendo a la búsqueda de las soluciones integrales correspondientes.

La meta de la acreditación transita por procesos intermedios, principios y compromisos. Estos procesos están periodizados como iniciales, intermedios y finales. En los procesos iniciales se encuentra la autoevaluación, siendo tarea prioritaria la adecuación de las matrices referenciales, que actualmente son dos: una de nuestra universidad y la otra de la Asamblea Nacional de Rectores. Es oportuno tomar la decisión de llevar a cabo la autoevaluación con fines de mejora.

La comunidad universitaria de psicología se compromete en mantener presente los principios de la calidad siguientes pertinencia, integridad, equidad, eficiencia, coherencia, adecuación y eficacia.

\section{Construir el perfil de competencias}

Tomar como referencia las competencias planteada por el proyecto Tuning, creando y manteniendo actualizados los perfiles estudiantiles y docentes, asumiendo el compromiso de construir las competencias específicas para la carrera de psicología.

Constituye un avance significativo la propuesta de perfil de competencias, planteado por el comité asesor de la Escuela Académico Profesional de Psicología, habiéndose convertido en el principal instrumento de trabajo en los términos de referencia correspondiente.

6. Crear espacios académicos que concreten las aspiraciones de la comunidad psicológica

El interés por el estudio de la vida psíquica de manera disciplinaria motivó la creación de la psicología como profesión en nuestra universidad. Convirtiéndose de esta manera en el primer espacio académico especializado de los estudios psicológicos en el Perú. 
En efecto, el 27 de abril de 1955, el consejo de Facultad de Letras aprueba el primer plan de estudios del naciente currículo universitario para la formación de psicólogos.

Acto histórico que se repite en otras universidades de Lima y del interior del país, llegando en el momento a ser 27 universidades que mantienen 38 programas de formación de psicólogos.

Panorama que debe responder a la diversidad de necesidades sociales existentes, donde la brecha de la desigualdad se siguen ahondando y en donde los problemas psicológicos exigen reparaciones urgentes.

Es tarea de la comunidad psicológica sanmarquina, compuesta por docentes, estudiantes y egresados, crear espacios académicos de investigación, intervención especializada e iniciativa profesional, para profundizar los estudios y configurar una práctica concreta para la búsqueda de soluciones integrales y compromiso activo.

\section{La reforma curricular una oportunidad y un desafío}

La última modificación del currículo de formación profesional de psicólogos se realizó en el año 1991, legalizando su implementación en 1995. El seminario curricular de 1999 concluyó que era necesario realizar una reforma consecuente, pero, por ausencia de decisiones oportunas, los cambios se han ido postergando año tras año, hasta que en el seminario curricular 2005 se realizó una evaluación curricular con un instrumento alcanzado por el vicerrectorado académico.

En este mismo año se crea una comisión Ad hoc encargada de redactar un documento base para la reforma curricular. Esta comisión estuvo presidida por el Mg. Alberto Quintana Peña, Director de la EAP de Psicología, y se concluye con la propuesta que ha servido como término de referencia para el debate del presente seminario curicular.

Hoy ante el avance acelerado de los cambios científicos y tecnológicos que repercuten en la conducta humana, incidiendo en la cognición, vida afectiva, socialización y estrategias de afrontamiento, la psicología debe explicar y promover modelos de investigación e intervención, requiriendo que las nuevas generaciones de psicólogos sean formados bajo nuevos parámetros de la ciencia psicológica.

8. El psicólogo en el Perú se desarrolla profesionalmente con ley propia

La reforma curricular parte de identificar a la psicología como una profesión que atiende la salud del comportamiento humano, principalmente en sus manifestaciones normales: El sustento legal de la psicología como profesión se encuentra legalizado en la Ley del trabajo del psicólogo No 28369 (28 de octubre de 2004) y en el Código de ética del psicólogo aprobado por el Colegio de Psicólogos del Perú. El mercado de trabajo está establecido en los siguientes ámbitos: educativo, salud, laboral, sociocultural, económico, recreativo y político.

\section{El marco doctrinario de la reforma se sustenta por principios y concepciones}

La reforma curricular orienta su actuación doctrinaria por principios y concepciones. Se asume plenamente los principios de los términos de referencia del comité asesor expresados en los siguientes: principio de la democratización de la educación, principio 
de la pertinencia contextual, principio de la coherencia interna, principio de la interdisciplinariedad, principio de la integración de competencias, principio de la significatividad del aprendizaje, agregando el principio de interculturalidad, la gratuidad de la enseñanza y el principio de valor de la responsabilidad social. Del mismo modo se asume plenamente las concepciones siguientes: concepción del ser humano, concepción de la salud, concepción de la educación, concepción de la psicología como ciencia, concepción de la psicología como profesión, concepción del aprendizaje, concepción de la enseñanza y concepción de la evaluación,

\section{La investigación orienta el compromiso}

La investigación se constituye como un eje formativo para desarrollar la sensibilidad del estudiante para detectar problemas y el hallar las soluciones requeridas. Razón suficiente para que los estudiantes reciban el asesoramiento y la orientación para la producción de conocimientos válidos $\mathrm{y}$, por lo tanto, el compromiso con la realidad nacional y el conocimiento científico.

El Instituto de Investigaciones Psicológicas anualmente aportará a la gestión curricular las prioridades de investigación que orienten el trabajo académico especializado para el entrenamiento en la investigación y para la realización de investigaciones con objeto de graduación y titulación profesional.

11. La reforma asume un currículo integral, de calidad, flexible, interdisciplinario y con pertinencia

El currículo es integral porque se organiza en base a las características esenciales de la universidad, trazando y orientando los requerimientos de la realidad nacional y los campos de intervención de la psicología, organizados y analizados por el paradigma de la complejidad

El modelo curricular reformado imprime una base humanística y científica en la formación del psicólogo, conforme está explicitado en los términos de referencia que el comité asesor de la EAP de Psicología propuso a este evento.

El currículo es de calidad porque está orientado por los atributos más desarrollados de la enseñanza y del aprendizaje, siendo trasparente en la rendición de cuentas al país y garantía para la excelencia académica y profesional.

El currículo es flexible porque permite al estudiante una formación integral y especializada, de acuerdo a los proyectos personales y profesionales, estableciendo un sistema de modificación y creación de nuevas áreas de desarrollo, de acuerdo a un sistema de reajuste y evaluación permanente, en seminarios curriculares anuales.

Técnicamente la flexibilidad se expresa en la matrícula de cursos obligatorios en los primeros años y electivos en los dos últimos años previos a las prácticas preprofesionales.

Los cursos obligatorios contienen los conocimientos indispensables que concretan los objetivos de los perfiles, pues estructuran las competencias que fundamentan la formación profesional del psicólogo, los mismos que deben ser aprobados por los estudiantes de manera directa, no sujetos a jurado ad hoc. 
Los cursos electivos orientan a la especialización del estudiante. El currículo reformado considera cuatro especialidades: Psicología Educativa, Psicología Clínica y de la Salud, Psicología Organizacional y Psicología Social Comunitaria; dejando abiertas otras áreas en desarrollo que se irán incrementando, progresivamente, de acuerdo a los recursos humanos de cuadros docentes y de intereses estudiantiles.

La matrícula en los cursos electivos se hará de una oferta amplia de asignaturas en cada una de las especialidades. Cada semestre ofrecerá tres o cuatro asignaturas, debiendo de elegirse una de ellas, decidida por el número de alumnos interesados. Para tal efecto, al concluir el semestre anterior, la EAP de Psicología convocará una prematrícula y la asignatura donde exista el mayor número de alumnos inscritos, será solicitada al Departamento Académico de Psicología para ser programada con el docente correspondiente. La dirección académica establecerá el número mínimo de alumnos inscritos para ser ofertada en el plan de estudios de matrícula.

Académicamente, la secuencia de las asignaturas no se basa en los prerrequisitos formales, excesivos y no académicos, sino en una seriación de conocimientos que transitan de una complejidad a otra complejidad.

Es responsabilidad del Comité Asesor de la EAP de Psicología establecer la secuencia de prerrequisitos.

El currículo es interdisciplinario porque permite el estudio especializado de disciplinas afines, sin las cuales no se podría entender en su integridad el fenómeno psicológico.

El currículo tiene pertinencia porque permanentemente se está actualizando de acuerdo a las necesidades del país.

\section{El diseño curricular}

El diseño curricular está compuesto por los siguientes componentes

a) Denominación del título profesional Psicólogo

b) Duración de la carrera 6 años

c) Carácter del currículo Flexible

d) Régimen del currículo Semestral de 17 semanas

e) Número de créditos total 300 créditos

\section{3. Áreas curriculares}

El currículo tendrá las siguientes áreas curriculares:

\section{a. Área de formación general}

En el currículo reformado se ubica en el primer año, que corresponde a los dos primeros ciclos semestrales, y tiene como objetivo fundamental orientar las expectativas académicas y profesionales que el estudiante tiene al ingresar a la universidad, es decir, brindar un conocimiento sistémico de la psicología como ciencia y como profesión. Al mismo tiempo introducirlo a un enfoque interdisciplinario de conocimientos de disciplinas afines, proveniente de la filosofía, 
de las matemáticas aplicadas (estadística), de las ciencias naturales y de las ciencias sociales, destacando el énfasis que se debe mantener en la antropología.

Con el objetivo de garantizar que los cursos respondan a las aspiraciones estratégicas de la reforma curricular, el departamento de psicología encargará a un profesor para que coordine las asignaturas provenientes de otros departamentos, teniendo como función principal realizar la gestión académica de la sumilla y la conducción de la enseñanza.

\section{b. Área de formación académica profesional básica}

En el currículo reformado se ubica entre el segundo y tercer año, que corresponde al tercero, cuarto, quinto y sexto ciclo, y tiene como objetivo profundizar el estudio del psiquismo humano en la complejidad de sus dimensiones, como son la personalidad, el desarrollo en todos sus aspectos, los procesos cognitivos básicos y superiores, los procesos afectivos y socialización, el estudio del cerebro, transitado del enfoque neurofisiológico a las neurociencias y en su construcción semiótica y las distintas propuestas de investigación.

Es importante señalar que la capacitación en este nivel no debe desconocer importantes aportes de la psicología básica que se expresa en los estudios de las modernas teorías del aprendizaje y la memoria, así mismo de la psicolingüística. Esta última debe mantenerse no solamente por su importancia, dada nuestra condición de ser país multilingüe, sino porque San Marcos fue la primera universidad que inauguró este tipo de conocimientos y posteriormente otras universidades la han seguido.

En esta área se estudia también las estrategias de evaluación psicológica y otras construcciones que hacen posible el registro-diagnóstico de hechos empíricos y no empíricos, orientados por los criterios de verdad que la ciencia moderna aporta para tipificar un conocimiento como científico.

En un tercer bloque de contextualizacion, se brinda un panorama de las distintas aplicaciones de la psicología, dejando abierta la posibilidad de integrar otros campos del conocimiento, como son la psicología dialéctica, psicología oncológica, psicología de emergencia y desastres, mercadotecnia, entre otras.

\section{c. Área mixta de formación Académica profesional básica y especializada}

En el currículo reformado se ubica en el cuarto y quinto año que corresponde al séptimo, octavo, noveno y décimo ciclo y tiene como objetivo combinar los estudios profundos de la psicología y sus aplicaciones especializadas, según las especialidades establecidas en la ley del trabajo del psicólogo de acuerdo a las siguientes especificaciones: áreas especializadas, área ética que es la matriz para el desarrollo profesional, área de estudios del futuro y áreas en desarrollo, las mismas que se precisan a continuación

\section{c.1. Psicología educativa}

El área de especialización en psicología educativa está orientada a capacitar al futuro psicólogo en las demandas de la educación de la realidad peruana, referida principalmente a la potencializacion de las capacidades a los estudiantes 
de los distintos niveles educativos, orientando el entrenamiento en la conducción de proyectos innovadores de apoyo a los proyectos curriculares y en las áreas de tutoría y orientación. Capacita también en problemas de aprendizaje, lenguaje, convivencia, clima emocional y motivacional, así como en programas inclusivos y en intervenciones preventivas, promocionales y terapéuticas.

\section{c.2. Psicología clínica y de la salud}

El área de especialización en psicología clínica y de la salud esta orientada a capacitar al futuro psicólogo en temas referidos principalmente a la relación individual y/o colectiva (casuística) que se establece entre el psicólogo y una persona que necesita ayuda especializada. La capacitación debe brindar los marcos conceptuales para que el profesional sea capaz de mejorar en la calidad de vida en la persona afectada en su comportamiento normal.

Es por ello que debe incidir en el conocimiento y el entrenamiento a un nivel básico especializado en competencias, habilidades, destrezas y actitudes requeridas y que deben estar explícitamente formulas en los perfiles universitarios, tales como competencias para la evaluación, diagnostico y tratamiento.

El tema central de esta área es la salud como fin y como tal la enseñanza y el aprendizaje debe nutrirse de los aportes teóricos de las distintas propuestas para intervenir en la multidimensionalidad que involucra la edad, el genero, cultura, familia, clase social y otros propios de la socialización humana.

Distinguir la enfermedad y la dolencia y especializarse en la segunda, dado el componente anímico e idiosincrásico compuesto por las implicancias históricas, sociales, culturales y psicológicas.

\section{c.3. Psicología organizacional}

El área de especialización en psicología organizacional esta orientada a capacitar al futuro psicólogo en temas referidos principalmente a la nueva cultura organizacional que aporta al mejoramiento de las relaciones humanas a través del impacto de una gestión productora de talentos y reconocimiento del capital humano. La capacitación en esta área permitirá que los futuros psicólogos tengan el conocimiento y entrenamiento en la gestión del cambio organizacional, recursos humanos, selección de personal,trabajo en equipo, manejo de conflictos y otros, propios del desarrollo de la especialidad.

\section{c.4. Psicología social comunitaria}

El área de especialización en psicología social comunitaria esta orientada a capacitar al futuro psicólogo en temas referidos principalmente al estudio de los procesos sociales desde la perspectiva psicológica. Estudia las representaciones sociales procesados por las personas, el papel de las clases sociales y las influencias de la historia y la cultura. Capacita al futuro psicólogo en el conocimiento y entrenamiento en proyectos de desarrollo comunitario.

En esta área hay que introducir modelos innovadores, los mismos que pasan por interesar a los estudiantes y docentes para cultivar este tipo de 
conocimientos. Tal es el caso de la propuesta de crear un taller de quechua, que debe ubicarse en la interculturalidad y en los estudios psicolinguisticos.

La Dirección de extensión universitaria y proyección social queda como responsabilizada para establecer los soportes académicos y profesionales de la promoción de experiencias innovadoras de la psicología social comunitaria.

\section{c.5. Área ética}

Es un área considerada como matriz para el desarrollo profesional del psicólogo. Cumple la función de orientar permanentemente la conducta moral de estudiantes, docentes y trabajadores. Promueve foros académicos y profesionales sobre los códigos de ética profesionales existentes en el mundo académico y profesional.

La gestión del área ética estará coordinada por la UNAYOE dentro de la estrategia del desarrollo sociopersonal y los proyectos personales de los alumnos. La meta es formar personas éticas, responsables consigo mismas, su profesión y el país.

\section{c.6. Área de estudios del futuro}

Es una área prospectiva de los estudios académicos y profesionales, haciendo gestión de los modelos sistémicos que explican los cambios acelerados en los referentes teóricos, practicos y tecnológicos que envuelve a los procesos en la actualidad. Promueve foros académicos y profesionales sobre las repercusiones en el bienestar y la calidad de vida.

El área de estudios del futuro deberá ser coordinada por el departamento académico de psicología, estableciendo las líneas estratégicas de desarrollo prospectivo de la ciencia psicológica.

\section{c.7. Áreas en desarrollo}

Es un conjunto de áreas de especialización que existen en la ley de trabajo del psicólogo y que en un tiempo corto se irán incrementando y desarrollando en el currículo universitario. Estas áreas son las siguientes psicología de las emergencias y desastres, psicología de la familia, psicología de las adicciones, psicología ambiental, psicología política, psicología jurídica, entre otras.

\section{d. Área de prácticas preprofesionales}

Tradicionalmente, el internado es la última fase de la formación profesional, periodo donde se evidencia el logro de las competencias de los perfiles universitarios. San Marcos tiene un espacio ganado en 86 espacios institucionales, donde los estudiantes realizan las prácticas preprofesionales. Estas son supervisadas y orientadas académicamente por un docente especializado. Es necesario que se actualicen los perfiles de competencias profesionales de dichas prácticas, al mismo tiempo que los perfiles de competencias docentes

El protagonismo del estudiante sanmarquino, que busca perfeccionarse permanentement, ha creado un espacio de prácticas, a las que ha denominado «prácticas libres», las cuales no cuentan con un reconocimiento formal en créditos, 
sin embargo, es necesario legalizarlas. La reforma curricular recomienda que éstas deben ser coordinadas por la Unidad de Asesoría y Orientación del Estudiante (UNAYOE).

\section{Uniformizar las sumillas de los cursos con criterios académicos explícitos}

Las sumillas de las asignaturas deben redactarse en base a los siguientes criterios:

Carácter del curso: téorico-práctico, colegiado-no colegiado.

Objetivos del curso: los que deben guardar perfecta coherencia con el perfil profesional y fundamentos del diseño curricular.

Síntesis del contenido.

Reseñas o referencias sobre la metodología a aplicar.

\section{Integrar progresivamente las nuevas tecnologías a la enseñanza}

Ante una generación estudiantil de la era digital, los medios y recursos tecnológicos de las TICs tienen que estar presentes en la enseñanza. En efecto, nuestra facultad cuenta con una sala de cómputo y los docentes tienen a disposición el equipamiento de multimedias directamente en las aulas, todavía de una manera móvil, por las previsiones de seguridad, pero atendidos casi en el $100 \%$ de aquellos profesores que manejan el diseño instruccional correspondiente. Del mismo modo, la universidad, desde hace varios años, tiene a disposición el aula virtual y, progresivamente, los docentes de psicología están haciendo uso de ello. De otro lado la revista de investigación en psicología desde el año 2005 se encuentra en la base de datos SISBI y muy pronto ingresará a otras bases de datos de dimensión internacional.

\section{REFERENCIAS BIBLIOGRÁFICAS}

1. Asamblea Nacional de Rectores (2005). Modelo de autoevaluación con fines de mejora de las carreras universitarias. Lima: DGIAU-ANR.

2. Benazic Tomé, Renato; Cuba Sancho Juana; García-Bedoya Maguiña, Carlos y Sánchez, Díaz Hugo (2006). Indicadores de gestión de la calidad de la Universidad Nacional Mayor de San Marcos. Lima: Oficina Central de Calidad Académica y Acreditación (OCCAA), UNMSM.

3. BM-UNESCO (2000). La educación superior en los paises en desarrollo. Peligros y promesas. Santiago de Chile: CPU.

4. Campos R. Manuel (2004). Fundamentación epistemológica y plan de estudios en la formación académica y profesional del psicólogo en la UNMSM. Lima: EAP de Psicología.

5. Centro Federado de Psicología (2005). Revista Psicología libre: Psicología, política y cultura Año I No 1, Año $2 \mathrm{~N}^{\circ}$ 1. Lima: CF de Psicología-UNMSM.

6. Comisión Transitoria del Seminario Curricular (2006). Informe del seminario curricular 2006. Lima: EAP de Psicología.

7. Decanato (2001). Gacetilla psicológica, Boletín del Decanato de la Facultad de Psicología. Lima: UNMSM. 
8. Delors, Jacques (1996). La educación encierra un tesoro. Madrid: Santillana-UNESCO.

9. Depaz, Zenón y Cuba, Juana (2003). Lineamientos para una política de calidad, autoevaluación y acreditación. Lima: Oficina Central de Calidad Académica y Acreditación (OCCAA), UNMSM.

10. EAP de Psicología (2006). Sistema curricular de de la Facultad de Psicología. Lima: Facultad de Psicología, UNMSM.

11. EAP de Psicología (2005). Seminario de evaluación y diseño curricular 2005. Lima: Facultad de Psicología, UNMSM.

12. EAP de Psicología (2005). Propuesta curricular 2005, Lima: EAP de Psicología

13. EAP de Psicología (1999). Informe final del seminario de evaluación del actual currículo de estudio. Lima: Facultad de Psicología, UNMSM.

14. EAP de Psicología (1996). Currículo de pregrado para la profesión de psicólogo. Lima: Facultad de Psicología, UNMSM.

15. El peruano. Ley 28740: Ley del sistema nacional de evaluación, acreditación y certificación de la calidad educativa. Lima, 2006.

16. El peruano. Ley 28369: Ley de trabajo del Psicólogo. Lima, 2004.

17. El peruano. Estatuto de la Universidad Nacional Mayor de San Marcos. Lima, 1984.

18. García A. Lupe (2003). «Creencias de control y autoeficacia en relación al rendimiento académico en estudiantes de la UNMSM». En Revista de Investigación en Psicología Año VI N ${ }^{\circ}$ 2. Lima: Instituto de Investigaciones Psicológicas, UNMSM.

19. García A. Lupe (2001). Informe: Prediagnóstico de la EAP de Psicología. Lima: Facultad de Psicología, UNMSM.

20. IPSI (1997). Investigación en Psicología: Retos para el futuro. Lima: UNMSM.

21. Montgomery, William (2004). Plan de estudios alternativo. Lima: EAP de Psicología.

22. Oficina Central de Calidad Académica y Acreditación (OCCAA) (2005). Hacia la autoevaluación en San Marcos. Lima: UNMSM.

23. Orellana, Oswaldo; Orellana, Daphne (2006) «Matriz de autoevaluación universitaria para la carrera de psicología». En Revista de Investigación en Psicología vol. $9 \mathrm{~N}^{\circ} 1$. Lima: Instituto de Investigaciones Psicológicas, UNMSM.

24. Orellana, Oswaldo (2003). «Reforma curricular en psicología». En Revista de Investigación en Psicología, Vol. VI, $\mathrm{N}^{\circ}$ 1. Lima: Instituto de Investigaciones Psicológicas, UNMSM.

25. Orellana, Oswaldo (2003). Proyecto curricular reformado de la Escuela académico Profesional de psicología. Lima: EAP de Psicología, UNMSM.

26. Orellana, Oswaldo (ed.) (2002). Boletín puertas abiertas. Lima: Facultad de Psicología, UNMSM.

27. Universidad Nacional Mayor de San Marcos. RR No 94610: Creación de la Facultad de Psicología. Lima: Secretaría General, 1988. 
28. UNESCO (2005). Una educación superior de calidad: Más allá de las fronteras. Lima: UNMSM.

29. Villegas, J., Marasi P. y Toro J. (2002). Problemas centrales para la formación académica y el entrenamiento profesional del psicólogo en las Américas. Chile: Universidad Central. 
$+1$ 